\title{
The Duration-distance Relationship and Average Envelope Shapes of Small Kamchatka Earthquakes
}

\author{
A. G. Petukhin ${ }^{1,2}$ and A. A. Gusev ${ }^{1}$
}

\begin{abstract}
Average envelope shapes (mean square amplitude time histories) of small earthquakes represent a convenient basis for the construction of semi-empirical stochastic "Green's functions," needed for prediction of future strong ground motion. At the same time, they provide crucial evidence for verification of the theories of scattering of high-frequency seismic waves in the lithosphere. To determine such shapes in the Kamchatka region we use the records of near $(R=50-200 \mathrm{~km})$ shallow earthquakes located around the broadband station PET. On these records, we select the $S$-wave group and determine its root-mean-square duration $T_{\mathrm{rms}}$, separately for each of the five octave frequency bands. We determine the empirical $T_{\text {rms }}$ vs. distance dependence and find it to be very close to a linear one. At the reference distance $R=100 \mathrm{~km}$, average $T_{\mathrm{rms}}$ decreases from $5.4 \mathrm{sec}$ for the $0.75 \mathrm{~Hz}$ band to $3.9 \mathrm{sec}$ for the $12 \mathrm{~Hz}$ band. To analyze average envelopes, we assume that the functional form of the envelope shape function is independent of distance, and stretch each of the observed envelopes along the time axis so as to reduce it to a fixed distance. Through averaging of these envelopes we obtain characteristic envelope shape functions. We qualitatively analyze these shapes and find that around the peak they are close to the shapes expected for a medium with power-law inhomogeneity spectrum, with the spectral exponent 3.5-4. From onset-topeak delay times we derive the values of transport mean free path and of scattering $Q$ for a set of distances.
\end{abstract}

Key words: Scattering, duration, envelope, Kamchatka.

\section{Introduction}

To reliably simulate destructive strong ground motion from a finite earthquake fault one needs the response of the medium to an elementary source. For relatively low frequencies, this problem can now be effectively solved with many modern techniques of calculation of theoretical seismograms. However, at high frequencies and reasonable distances (say, above $2 \mathrm{~Hz}$ at $30-\mathrm{km}$ distance or farther), the application of these deterministic techniques meets with difficulties; one of them is that they require very detailed and often unavailable information about the structure of earth medium.

\footnotetext{
${ }^{1}$ Institute of Volcanic Geology and Geochemistry RAS, 9 Piip Blvd., Petropavlovsk-Kamchatsky, 683006, Russia. E-mail: gusev@emsd.iks.ru

${ }^{2}$ Now: Geo-Research Institute, 4-3-2 Itachibori, Nishi-ku, Osaka, 550-0012, Japan. E-mail: anatoly@geor.or.jp
} 
Another possibility is to use observed small earthquake records as "empirical Green's functions" (EGF). This approach, though attractive, is far from being universal. Usually its applicability is demonstrated using aftershocks, whereas it may well be impossible to find records of small earthquakes with hypocenters and fault plane solutions sufficiently close to those of a target potential earthquake fault. Another complication is the significant variability of small earthquake records that creates a problem of choosing the "right" EGF. At last, EGF are highly sitedependent; this is an advantage when a particular site needs assessment, however inconvenient when a more general description of potential earthquake effects is sought for (say, for a long lifeline or a broad residential area).

As an alternative to these deterministic approaches, one may treat the highfrequency (HF) part of the record as a random function. A standard stochastic description is to combine a "modulating" envelope function (positive, non-stationary but slow-varying) and stationary noise-like "carrier" high-frequency signal. The envelope function of maximum amplitudes on the record (that is, $S$-wave group) is usually pulse-like, and can be considered as the instant mean square amplitude of the signal. The noise-like "carrier" is a random signal, and may be taken to have unit variance. It is this stochastic approach that we deal with in the present study.

Recently a combined approach was put into practice by KAMAE et al. (1998). They simulated strong ground motion using a hybrid Green's function technique: the long-period motions from a small event were deterministically calculated using the 3-D finite-difference method, whereas the high-frequency motions were stochastically simulated using BoORE's (1983) method.

One can argue (HADDON, 2000) that the high-frequency seismic record consists of particular arrivals that may be effectively predicted theoretically on the basis of a layered or more complicated model, and thus the stochastic approach, at least with respect to propagation effects, is redundant and misleading. This may be partly true in particular cases, in the first place in continental areas where a short $L_{g}$ phase is often dominating in the $S$-wave group. The usefulness of the stochastic approach, as usual, can be proved only by its efficiency. At relatively low frequencies, and in locations with well-known structure, especially when the deviations from the layered earth model are slight, the deterministic approach is adequate. At higher frequencies, with complex structures, stochastic approach often becomes the only workable one. For example, to predict effects of an $M=8$ event in a geologically complex area (such as island arc or mountain system), at epicentral distances in excess of $25 \mathrm{~km}$ and at frequencies above $1-3 \mathrm{~Hz}$, the stochastic approach has no real alternatives.

The description of earthquake signals through envelope and stationary carrier is well known among seismologists and earthquake engineers, most often for the case of moderate-to-large earthquakes. The most important feature of the envelope function is its duration defined in some convenient way. TRIFUNAC and BRADY (1975) found that both source process and propagation in the medium contribute 
significantly to the duration of an accelerogram (which represents a most common HF signal, and is mostly caused by the $S$-wave group). The path-related contribution to duration increases with epicentral distance; moreover, it is approximately proportional to it. The evident explanation for such a behavior is the common dispersion of the surface-wave train: it must cause just this picture. This explanation is probably too simplistic however because the well-known surface wave signature is a deterministic dispersive wave-train with good spatial coherence, whereas the observed $S$-wave group is noise-like and usually has a correlation distance of the order of the wavelength. The models of common waveguides with scatterers (MALIN, 1980; WANG and HerrmanN, 1988) or of random waveguides (LEARY et al., 1993) seem to be considerably more relevant, but they are not sufficiently advanced at present to be directly compared to data.

On the other hand, one may treat the $S$-wave group as a body-wave phenomenon. Depending on the character of real data, one may prefer a deterministic description (through multiple rays) or a stochastic one, which seems to be more appropriate for our observations. Initially we attempt to describe observations in a phenomenological fashion, through determining average envelope shapes and then through estimating their dependence on frequency and distance. Then we try to explain the results in frames of a particular model, namely that of random forward scattering. This particular model, with some additional assumptions, predicts more or less realistic envelope shape (Williamson, 1972; GuSEv and ABubakirov, 1996). In frames of this model, the distance dependence of the duration of the envelope may be employed for determination of the scattering parameters of the lithosphere (GUSEV and Lemzikov, 1983, 1985; SAto, 1989; Gusev and Abubakirov, 1999a,b). There is a problem however: the duration vs. distance behavior predicted by Williamson's theory often does not match the observations well. Indeed, Williamson's theory predicts that duration is proportional to distance squared, in clear contradiction, e.g., to Trifunac and Brady's result. In one case this contradiction was successfully resolved. For nearly-vertically propagating rays, an approximately linear increase of duration with distance can be consistently explained by the strong vertical nonuniformity of the scattering coefficient (GUSEV and ABUbAKIROv, 1999a,b). However, for wave propagation from crustal sources at distances exceeding $50 \mathrm{~km}$, this explanation cannot be directly applied.

We are unaware of any theory that might consistently explain the linear increase of the duration of the HF $S$-wave group with distance. (This duration should not be confused with the duration of the first pulse, or half-wave.) On the other hand, the idea that this increase is indeed so close to linear was never verified accurately: strong motion data cannot resolve the accurate mode of the duration vs. distance relationship. Thus, it is interesting to establish this relationship with a certain accuracy and in a systematic manner. The results of such a study may serve as solid input information for semi-empirical strong-motion simulations; simultaneously they would represent a well-defined piece of evidence that may be used for validation of 
any future advanced theory of random wave propagation in the lithosphere. For similar reasons, accurate determination of envelope shapes also deserves attention.

For the problem of determination of the average envelope shape, a significant complication is the need to estimate the empirical function of two variables: time and distance. To circumvent this problem we will assume that the envelope shape is functionally the same over a wide distance range. That is, the mean observed envelope can be written approximately as $F\left(t / t^{*}\right)$ where $t^{*}$ is a distance-dependent constant, and $F()$ is a universal function, one for all distances. Any reader acquainted with short-period seismograms of near earthquakes knows that it is (almost) impossible to estimate the epicentral distance from the sole appearance of a record with an unknown "paper speed," and this impossibility just means that the universal envelope function is a sound idea. Incidentally, many theoretical envelope shape functions are also distance-independent. In both cases, the envelopes obtained at different distances will match after appropriate squeezing or stretching of the time axis.

The data analysis was performed in the following order. First we select threecomponent digital records, separate $S$-wave groups and find the duration parameter for each component. We use the systematic definition of duration through the second temporal moment of squared amplitude. To account for frequency dependence of durations and envelopes, this is done separately for each of the six filtered traces covering the $0.5-16 \mathrm{~Hz}$ range, five with one-octave bandwidth, and one wideband. We analyze the duration vs. distance dependence and find that it is well described by a simple linear proportionality. Then we stretch the time axis of each envelope so as to reduce it to a fixed distance, and we average all envelopes for a given frequency band, to obtain stable and reliable empirical averages. Lastly, we compare these envelopes with theoretical shapes and determine apparent parameters of scattering including estimation of scattering attenuation.

\section{Method}

\section{Rms Duration and Other Definitions of Duration}

A number of definitions of duration of high-frequency earthquake signal exist among seismologists and earthquake engineers. One popular idea (APTIKAEV, 1975; KAWASHIMA et al., 1985) is to depart from the peak value and to treat as significant the segment of the time axis in which the amplitudes are in excess of some fraction (say, $50 \%$ ) of the peak value. The duration parameter $T_{0.5}$ is then defined as the duration of the significant part of the signal. Another approach is to integrate the power (squared amplitude) of the record, take the moments when this integral equals to, say $5 \%$ and $95 \%$ of its final value, and calculate duration $\left(T_{90}\right)$ as the interval between these marks, containing $90 \%$ of total energy (TRIFUNAC and BRADY, 1975). 
GuSEV (1983) proposed the use of rms duration $T_{\mathrm{rms}}$ defined through the normalized second central moment of squared amplitude $A^{2}(t)$ :

$$
T_{\mathrm{rms}}^{2}=e_{2} / e_{0}-\left(e_{1} / e_{0}\right)^{2},
$$

where $e_{j}$ are initial power moments over time:

$$
e_{j}=\int_{0}^{\infty} t^{j} A^{2}(t) d t \quad(j=0,1,2) .
$$

If one treats the squared amplitude as probability density, then $T_{90}$ and $T_{\mathrm{rms}}^{2}$ correspond to the $5 \%-95 \%$ inter-quantile range and variance, respectively.

The justification for one more definition is its very attractive theoretical properties. Earthquake signals can be often treated, at least approximately, as the convolution of source-related and path-related components. This is of course also true for their extracted HF part. For the high-frequency part of signals of stronger earthquakes recorded at longer distances in heterogeneous structures (say, $f>1 \mathrm{~Hz}$ signal components of $M>6$ events recorded at $R>30 \mathrm{~km}$ in an island arc), both the source and the path contributions have a stochastic, noise-like character. It is not widely known that in this case the envelope (mean square amplitude) of the convolved signal approximately coincides with the convolution of envelopes of components. (This fact is well known in radiophysics and laser optics, see e.g., IsHIMARU (1978); the entire field of lidar data analysis is based upon it). In seismology it was introduced by Rautian (1976) and KopNichev and ShPiLKer (1978). As the second central moment of convolution is the sum of the second central moments of components (in probabilistic terms, the variance of sum is the sum of variances of components), then (GUSEV, 1983)

$$
T_{r m s, \text { total }}^{2}=T_{r m s, \text { source }}^{2}+T_{r m s, \text { path }}^{2},
$$

where $T_{r m s, \text { source }}$ is the rms duration of squared HF source wavelet for a ray to a given receiver, $T_{r m s, \text { path }}$ is the rms duration of squared average Green's function of the path, and $T_{r m s, \text { total }}$ is the rms duration of the recorded signal. Therefore, by using rms duration definition, the problem regarding how to combine source and path effects on duration acquires a simple, intuitively transparent and theoretically wellgrounded solution. In particular, equation (3) provides a ready structure for a relevant empirical formula. A simple way to determine $T_{r m s, \text { path }}$ empirically is to approximate it by $T_{r m s, \text { total }}$ of a small earthquake; this is justified by the fact that the source time function of a small earthquake is sufficiently near the $\delta$-function.

\section{Determining rms Duration in Practice}

An important issue in the practical determination of $T_{\mathrm{rms}}$ on the basis of eqs. (1) and (2) is the choice of the time window for the integration (or, for the $S$-wave 
group). Its beginning creates no problem, but for the selection of its end there is no natural rule. Furthermore such a rule is needed because coda decays slowly thus making $T_{\text {rms }}$ estimates somewhat dependent on the choice of the window. The results of TRIFUNAC and BRADY (1975) suggest that the length of the window can be varied in proportion to distance. This idea also agrees with our assumption of a standard envelope shape. Therefore, we set the length of the window proportional to the $S$ - $P$ interval, with the particular value of coefficient (2.0) that was further selected on the empirical basis.

Data processing is carried out in the following manner. First, we filter each record by means of five 3rd-order one-octave $(0.5-1,1-2,2-4,4-8$, and $8-16 \mathrm{~Hz})$ Butterworth filters in direct and inverse time. Also a wide band $(0.5-16 \mathrm{~Hz})$ filter was used. $P$ and $S$ onsets are picked from displacement records. When an $S$-onset pick was doubtful, the calculated $S$-onset time was used instead. Then the $S$-wave window is defined, with the length proportional to the $S-P$ interval. Over this window, integration (2) of squared amplitude was performed. To stabilize the result, we combine the signal $x(t)$ with its Hilbert transform $H\{x(t)\}$ and use the square of the modulus of the analytical signal as the squared amplitude:

$$
A^{2}(t)=x^{2}(t)+H^{2}\{x(t)\} .
$$

This procedure was further modified to eliminate the bias (sometimes significant) related to microseismic noise. Assuming signal and noise to be uncorrelated, one can write down the corrected squared signal amplitude as:

$$
A_{s}^{2}(t)=A_{s+n}^{2}(t)-\overline{A_{n}^{2}},
$$

where $A_{s+n}(t)$ is the "raw" recorded (filtered) signal, and $\overline{A_{n}^{2}}$ is the mean square amplitude of the noise. Noise is assumed stationary, thus its squared amplitude is constant. In practice, $\overline{A_{n}^{2}}$ is estimated as the average squared noise from a record segment before the $P$ onset. This procedure was performed for each frequency band.

\section{Duration vs. Distance Relationship}

Now we pass to the procedures for determination of the $T_{\mathrm{rms}}$ vs. distance dependence. After the work of TRIFUNAC and BRADY (1975) and other studies in this line one can expect that the $T_{\text {rms }}$ vs. epicentral distance dependence for shallow earthquakes will be close to a linear one. Contrastingly, from Williamson (1972) and SATo (1989) one can expect the duration to increase as the 1.5-2 power of distance. For this reason we apply the following formula to our data analysis:

$$
T_{\text {rms }}=T_{\text {ref }}\left(R / R_{\text {ref }}\right)^{n},
$$

where $R_{\text {ref }}$ is the fixed reference distance, taken further as $100 \mathrm{~km}$, and $T_{\text {ref }}=T_{100}$ and $n$ are the unknowns to be fit to the data. 


\section{Determination of Envelope Shapes}

With the $n$ value at hand, we can pass to the estimation of the envelope shape. This can easily be done for any fixed $n$; but using the fact, to be settled further, that the optimal $n$ value is very near 1.0 , we confine ourselves to the case $n=1$. The assumption of the standard shape function means that

$$
\overline{A^{2}(t)}=c F\left(t / t_{c}\right)
$$

where $t_{c}=t_{c}(R)$ is the characteristic duration. With $t_{c}$ proportional to $R$, this yields $\overline{A^{2}(t)}=c F\left(t / t_{p}\right)$ where $t_{p}$ may represent, e.g., the $S$-wave travel time for a particular record. To estimate the $F(\tau)\left(\tau=t / t_{p}\right)$ function from the data, we need to compress or expand the time axis. Technically, with the data in the form of a sequence of samples with a constant time step, it is sufficient to change this step. Then, for convenience in further data analysis, thus modified data are resampled (by interpolation) with a common time step. The envelope of each modified record is smoothed; and at last we decimate the result. This allows us to limit the number of points where the $F(\tau)$ is determined, without sacrificing significant details. The resulting sequence is then normalized to its own "energy" $e_{0}$.

To find the characteristic shape, all normalized envelopes were averaged over all available traces; and the result was smoothed again.

\section{Initial Data and the Study of RMS Durations}

\section{Data}

Initial data are the records of the broadband digital station PET, installed at Petropavlovsk-Kamchatsky, Kamchatka, Russia, in September 1993. The station is located on a hard rock mass and its possible site effect on the duration is negligible. To study the rms duration we used the records of the short-period (SP) channel with a sampling frequency of $80 \mathrm{~Hz}$, obtained in 1993-1994. We selected events with depths $0-40 \mathrm{~km}$ and distances extending to $200 \mathrm{~km}$. In order to obtain representative results, we wanted to evenly cover the analyzed distance range. We used all available records for the distance range $0-80 \mathrm{~km}$. Some data for the $80-200 \mathrm{~km}$ range were rejected in a random manner. As a result we obtained about 15 records per every 20-km distance interval. To guarantee sufficiently short source duration, events with magnitudes exceeding 4.5 were excluded. As is known for the region in question, the duration of the $S$-wave group significantly depends on the source depth (ABUBAKIRov and Gusev, 1990; Gusev and Abubakirov, 1999b). This governed the selection of only shallow events $(H<40 \mathrm{~km})$. Most source depth values are between 20 and $40 \mathrm{~km}$ (with some tolerance), whereas the Moho depth here is between 25 and $35 \mathrm{~km}$. Thus, most events cannot be considered as definitely crustal or definitely subcrustal. 


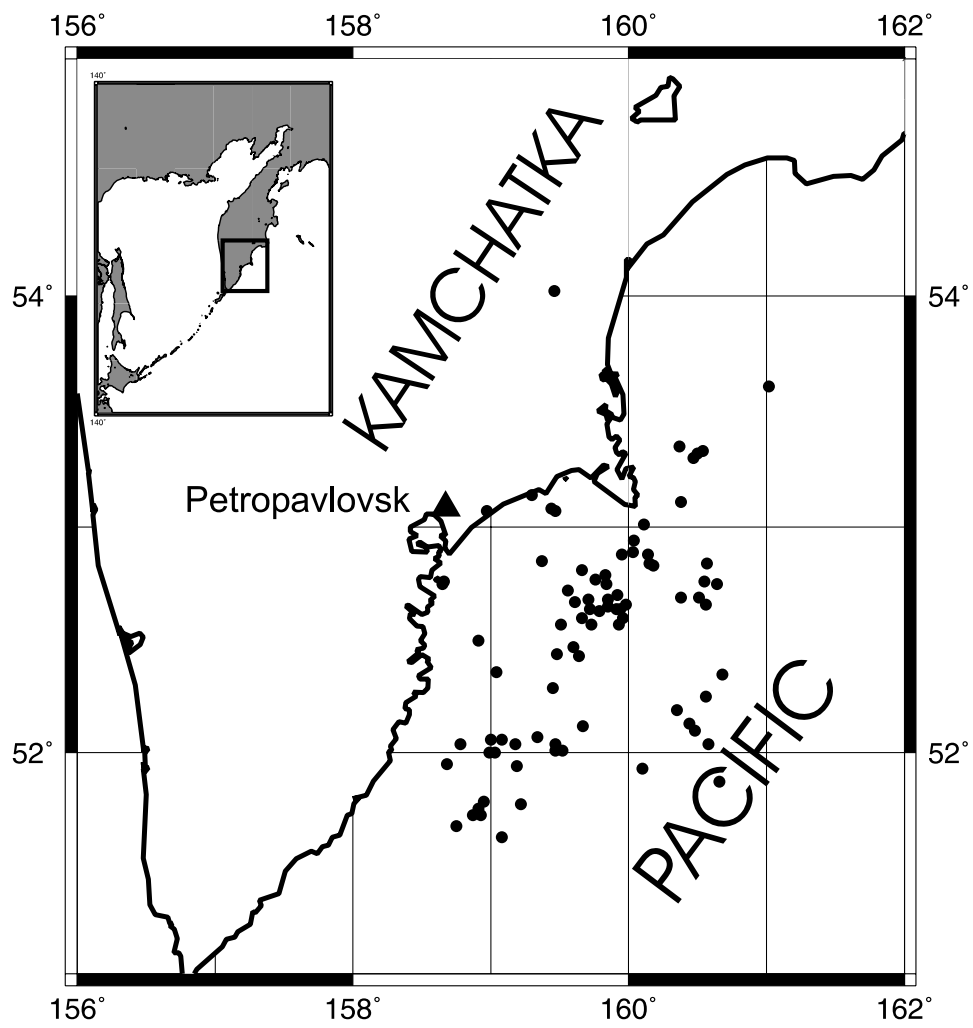

Figure 1

Epicenters of the earthquakes recorded by the IRIS station Petropavlovsk and used in this paper.

The final data set includes three-component records of 82 earthquakes; their epicenters are mapped on Figure 1. The appearance of the $S$-wave group within the seismic record was random-like. No systematic arrivals suggestive of $L_{g}$ or Moho reflection are known for station PET as well as for other stations on the eastern coast of Kamchatka. Thus, the interpretation of these data in a stochastic manner evidently was a reasonable idea.

\section{Estimating $T_{\mathrm{rms}}$}

The selected data set was processed according to the procedure described in the previous section. Firstly, velocity records of SHZ/SHN/SHE channels (80 sps) were converted to acceleration (in view of a possible engineering application) and then bandpass filtered. An example of a filtered component is shown in Figure 2. Then we had to set the integration window. To do this in a systematic manner, we had to choose the value of the coefficient $K$ in the equation:

$$
\Delta t=K\left(t_{S}-t_{P}\right),
$$




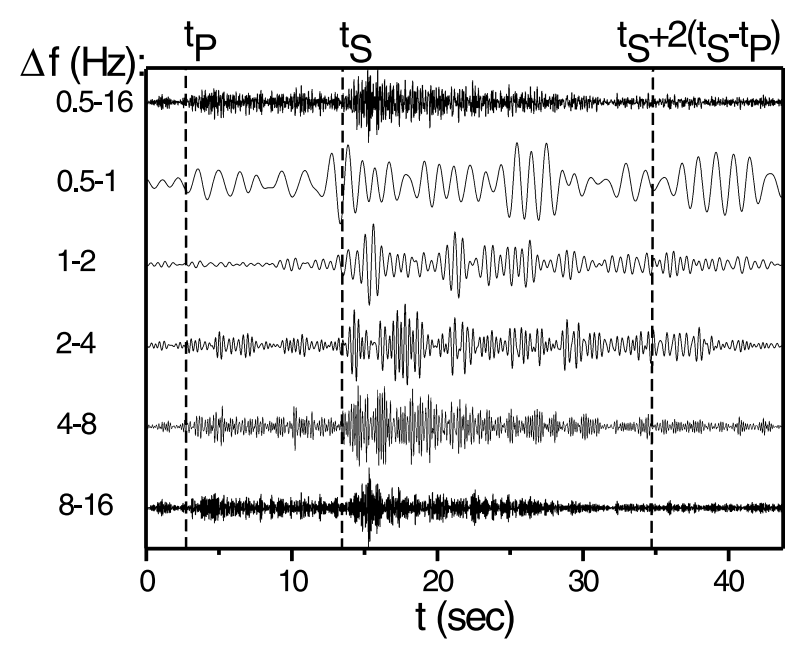

Figure 2

Example of a small earthquake record (acceleration) filtered into 5 one-octave frequency bands $(0.5-1,1-2$, 2-4, 4-8 and 8-16 Hz) and into the wide $(0.5-16 \mathrm{~Hz})$ band. Vertical lines show $P$ and $S$ arrivals and also the end of the time window which was used to cut out the direct waves for the case of $K=2$.

where $\Delta t$ is again the width of the time window for integration in (2) and $t_{S}-t_{P}$ is the $S$ - $P$ interval. In Figure 3 we see how the rms duration (averaged over a particular distance range) depends on the choice of $K$. We show the result for the wide-band channel; for the other bands the results are similar. One can see that in each distance range, the average rms duration increases with increase of $K$, as could be expected, but that this increase nearly saturates at $K=2$. On this basis, we selected $K=2$ for the further processing. Then the $T_{\text {rms }}$ values were calculated.

\section{Analysis of the $T_{\mathrm{rms}}$ vs. Distance Trend}

In Figure 4 we see the observed $T_{\text {rms }}$ vs. distance trends for all frequency bands in the log-log scale. We see quite stable trends, visually near linear, in agreement with our assumed equation (7). To determine $T_{\text {rms }}$ and $n$, we now perform linear regression according to the equation:

$$
y=a+b x+\epsilon
$$

where $y=\log \left(T_{\mathrm{rms}}\right), x=\log (R / 100), \epsilon$ is a random error, and unknowns $a$ and $b$ are $\log \left(T_{100}\right)$ and $n$, respectively. The results of the fit by least squares are shown in Table 1.

Most estimated $n$ values are insignificantly different from 1.0. Therefore, the usual assumption $n=1$ (TrifunaC and Brady, 1975; RAUTIAN and Nikiforova, 1980) can be applied to Kamchatka data with certainty. The $T_{100}$ value $\left(T_{\mathrm{rms}}\right.$ for 


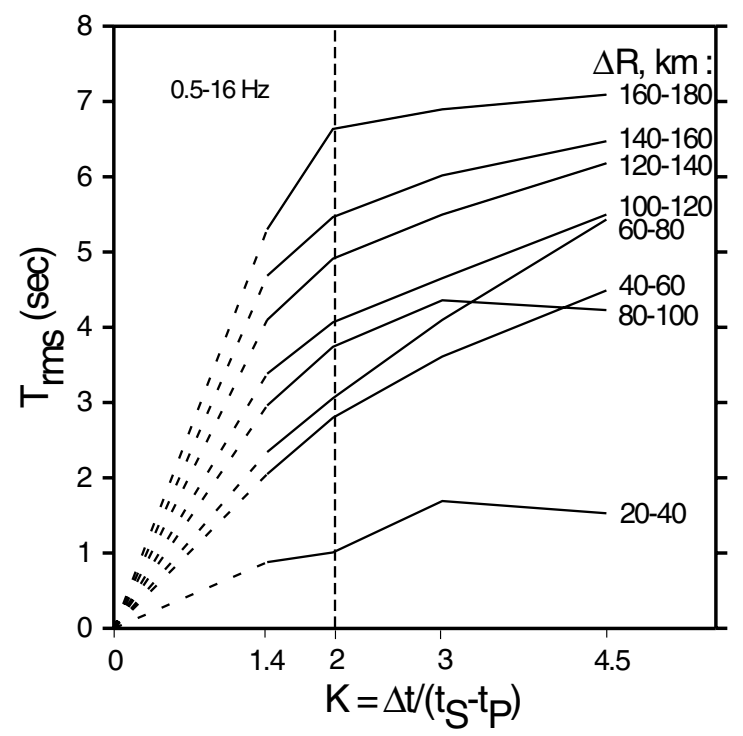

Figure 3

The relationship between $T_{\mathrm{rms}}$ and the length of the direct $S$-waves time window for all distance range bins. The vertical dashed line indicates the window length used to estimate average parameters in this paper.

$R=100 \mathrm{~km}$ ) is also listed in Table 1 . Note that the scatter of individual $T_{\mathrm{rms}}$ values is moderate: $\sigma(\epsilon)$ is about 0.1 , meaning that at a given distance, typical $T_{\text {rms }}$ variations for a particular component are between $80 \%$ and $125 \%$ of the average. Individual $T_{\text {rms }}$ estimates for various components are positively correlated, and the degree of correlation increases with frequency. We cite here the coefficient of correlation between a horizontal component and the vertical component (average over EW-Z and NS-Z pair sequences); it equals to $0.11,0.47,0.76,0.80,0.74$ and 0.83 for $\Delta f=$ $0.5-1,1-2,2-4,4-8,8-16$ and $0.5-16 \mathrm{~Hz}$ respectively. In Figure 5 one can see the frequency dependence of $T_{100}$. The set of estimated $T_{100}$ and $n$ values represents the first main result of the present study.

It is interesting to compare the $T_{100}$ estimates between various frequency bands and also between horizontal and vertical components (see Table 1 and Fig. 5). With increasing frequency, rms envelope durations decrease at all components. On average, $T_{100}$ decreases from $5.5 \mathrm{sec}$ at the $0.5-1 \mathrm{~Hz}$ band to $4.0 \mathrm{sec}$ at the $8-16 \mathrm{~Hz}$ band. The increase of duration at lower frequencies agrees with the appearance of the wideband $S$ waveforms: their later parts have (visually) considerably lower frequencies as compared to the part just after the $S$ onset. The duration of the $S$-wave group on the vertical component is $5-9 \%$ larger than on the horizontal one. This effect is more pronounced in the higherfrequency bands. 

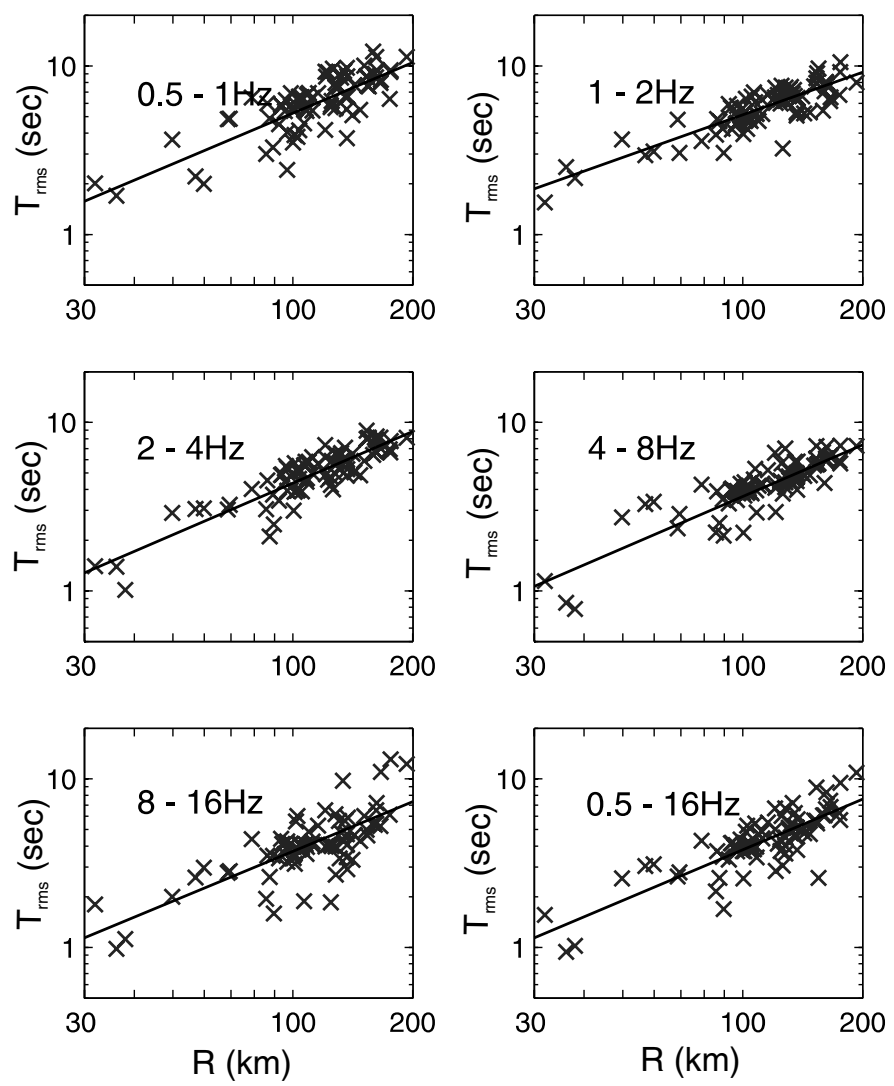

Figure 4

Distance dependence of $T_{\text {rms }}$ for the case of the direct $S$-waves window defined by the value $K=2$, the average estimate over horizontal components, frequency bands: $\Delta f=0.5-1,1-2,2-4,4-8$, $8-16$ and $0.5-16 \mathrm{~Hz}$.

\section{Determination and Analysis of Average Envelopes}

The second part of the work deals with the determination of average empirical envelopes of $S$-wave groups of small earthquakes on the horizontal component. With this aim we processed the same records as used for the $T_{\text {rms }}$ study, using the procedure described above. The only deviation from the ideal procedure is related to limited data quality. When the equation (5) was applied, one or more segments of a particular envelope could sometimes become negative (local estimate of the "signal + noise" level is below average noise). This situation indicates that the local estimate of signal power is unreliable, and hardly will become reliable again later along the record. For this reason, only one continuous segment of the corresponding record (that contains the maximum amplitude) was kept for further processing. Some traces of lower-frequency bands were discarded completely. Thus 
Table 1

Parameters of the regression equation $\log T_{\mathrm{rms}}=\log T_{100}+n \log (R / 100)+\varepsilon$. Linear regression (equation (9)) is performed for observed $T_{\mathrm{rms}}$ data calculated for the case $K=2$. $\varepsilon$ is random error. In calculations for "horizontal" component, the average $T_{\mathrm{rms}}$ over EW and NS components was used.

\begin{tabular}{cccccc}
\hline$\Delta f, \mathrm{~Hz}$ & $T_{100}$ & $\sigma\left(\log T_{100}\right)$ & $n$ & $\sigma(n)$ & $\sigma(\varepsilon)$ \\
\hline & & Horizontal component & & \\
$0.5-1$ & 5.37 & 0.18 & 1.0 & 0.09 & 0.12 \\
$1-2$ & 4.96 & 0.12 & 0.97 & 0.06 & 0.08 \\
$2-4$ & 4.27 & 0.14 & 1.01 & 0.07 & 0.09 \\
$4-8$ & 3.72 & 0.14 & 0.94 & 0.07 & 0.13 \\
$8-16$ & 3.76 & 0.21 & 0.92 & 0.10 & 0.12 \\
$0.5-16$ & 3.80 & 0.18 & 0.94 & 0.09 & 0.11 \\
$0.5-1$ & & Vertical component & & 0.07 \\
$1-2$ & 5.62 & 0.17 & 1.00 & 0.08 & 0.08 \\
$2-4$ & 5.01 & 0.10 & 0.99 & 0.05 & 0.08 \\
$4-8$ & 4.37 & 0.12 & 0.95 & 0.06 & 0.13 \\
$8-16$ & 4.07 & 0.13 & 0.90 & 0.06 & 0.14 \\
$0.5-16$ & 4.27 & 0.20 & 0.92 & 0.10 & \\
\hline
\end{tabular}

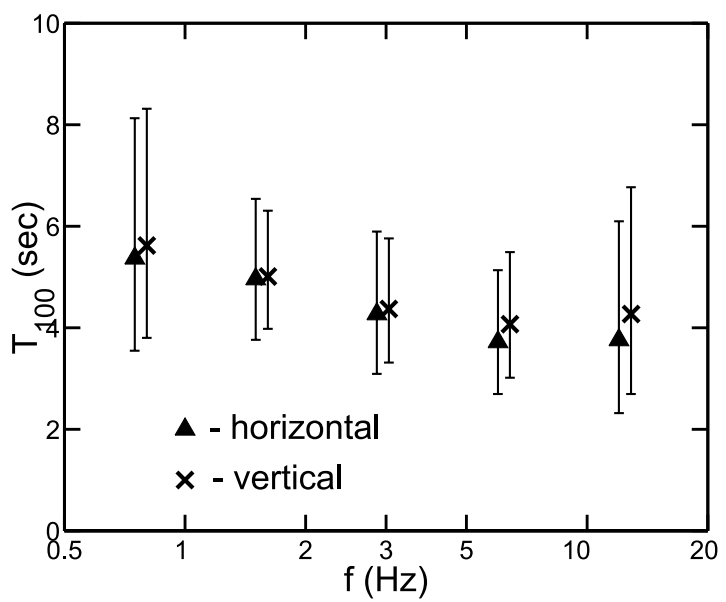

Figure 5

Frequency dependence of the $T_{100}$ parameter for horizontal (average over NS and EW) and vertical components (see also Table 1).

the data volume and so the accuracy of our envelope estimates deteriorate at the ends of the time segment and also at lower-frequency bands.

After passing this check, the smoothed data were modified by the abovementioned procedure for expansion/contraction of the time axis. For this procedure, the reference distance of $200 \mathrm{~km}$ was used, so that actually all envelopes were only expanded. After expanding and normalization, the individual envelopes were 


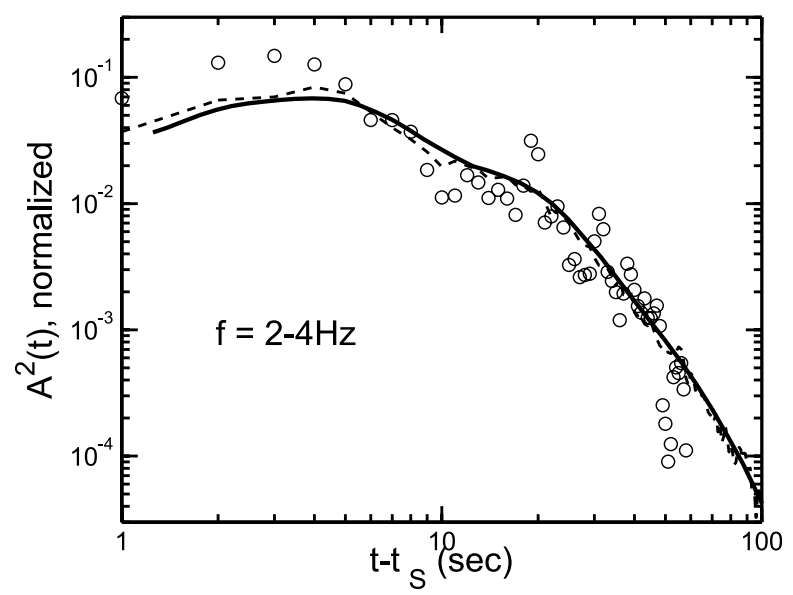

Figure 6

Example of calculation of average envelope, $\Delta f=2-4 \mathrm{~Hz}$. Circles: the "raw" envelope of an example individual record reduced to the distance $R=200 \mathrm{~km}$; dashed line: envelope, averaged over individual envelopes reduced to $R=200 \mathrm{~km}$ and smoothed with constant window $\Delta T=2 \mathrm{sec}$; solid line: the final average envelope for $\Delta f=2-4 \mathrm{~Hz}$.

averaged and smoothed again to produce the estimates of mean envelope shapes. Figure 6 illustrates the applied procedure for the frequency band 2-4 Hz. The resulting envelopes can be seen on Figure 8 in the linear scale and on Figure 7 in the $\log -\log$ scale. Both error bounds for average envelopes and the scatter of individual envelopes are represented graphically on Figure 7. Accuracy varies for the reasons discussed above; in the worst case we have a point based on only 7 individual data, against 164 for the best points.

These envelopes represent the second main result of the work; they are ready for application in strong-motion modeling by stochastic approach.

The shape of average envelopes is generally consistent with the shape of an envelope scattered in the random medium (WILLIAMSON, 1972): they have the typical steep leading edge and gradually decreasing trailing edge. One remarkable feature is the second hump at the delay time $15-30 \mathrm{sec}$ that is seen for the $0.5-1 \mathrm{~Hz}$ band. A similar but less pronounced tendency is also clearly seen for $1-2 \mathrm{~Hz}$ band (compare Figure 7 and Figure 8) and also may be noted for 2-4 Hz band. This feature can be tentatively associated with the arrival of a "slow" train of short-period surface waves with group velocities between 3.5 and $2.8 \mathrm{~km} / \mathrm{s}$. The average delays $t_{m}$ for maxima of $S$-wave envelopes with respect to $S$-wave onsets (onset-to-peak delays) are given in Table 2. Between 0.5 and $8 \mathrm{~Hz}$ they clearly increase with frequency. This result is seemingly in contradiction with the already ascertained fact that $T_{\mathrm{rms}}$ values decrease with frequency. This difference is caused by the presence of the mentioned "second hump" feature in the tail parts of lower-frequency envelopes. Although the 


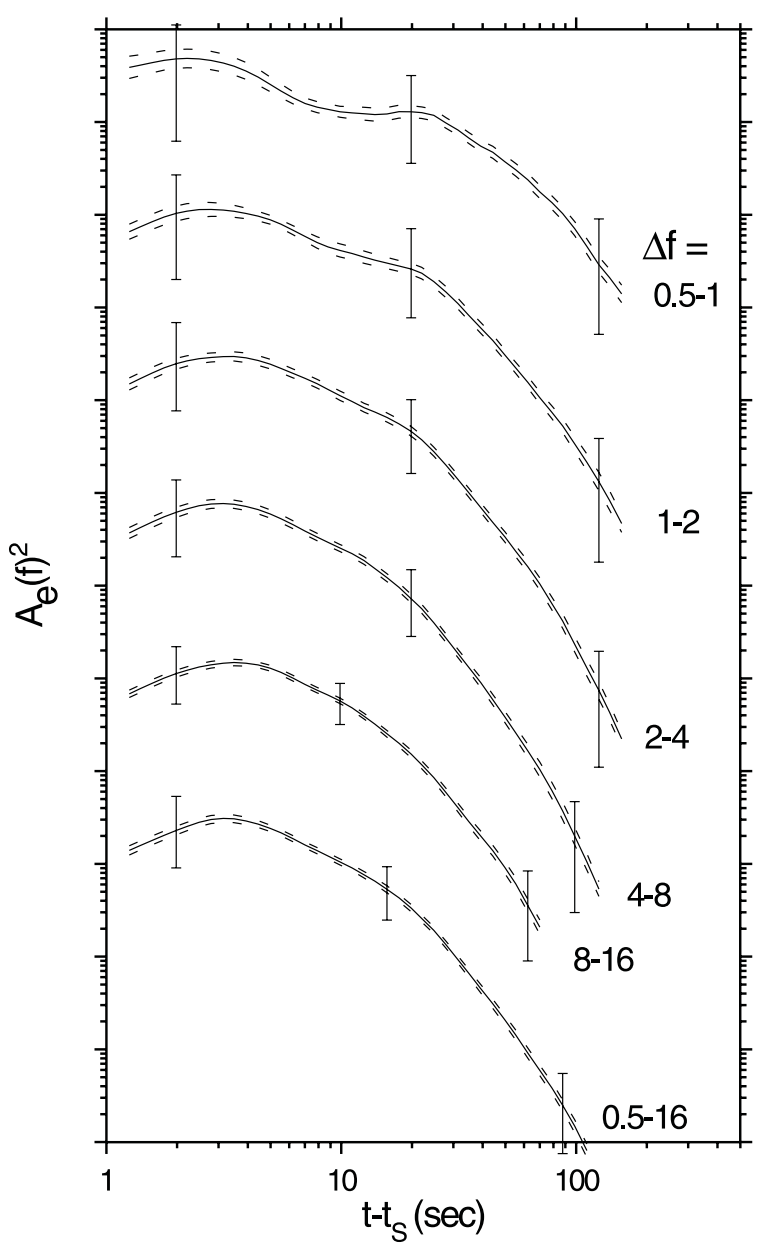

Figure 7

Averaged smoothed envelopes of filtered records in log-log scale. Dashed lines represent the $\pm 1 \sigma$ corridor for the estimated average envelope; error bars show the $\pm 1 \sigma$ scatter of individual data points. Envelopes have normalized amplitudes and, for clarity, are shifted in a vertical direction by two decades.

amplitude of the "hump" is not large, its contribution to the value of $T_{\mathrm{rms}}$ is greatly enhanced by the $t^{2}$ weighting in the integration (2).

\section{Estimating Apparent Mean-free Path and Scattering Attenuation}

We can now compare the observed average envelope shapes (we shall also refer to them as "pulse shapes") with theoretical ones. One relevant shape function was calculated by WILLIAMSON $(1972,1975)$ for the case of forward scattering of energy 


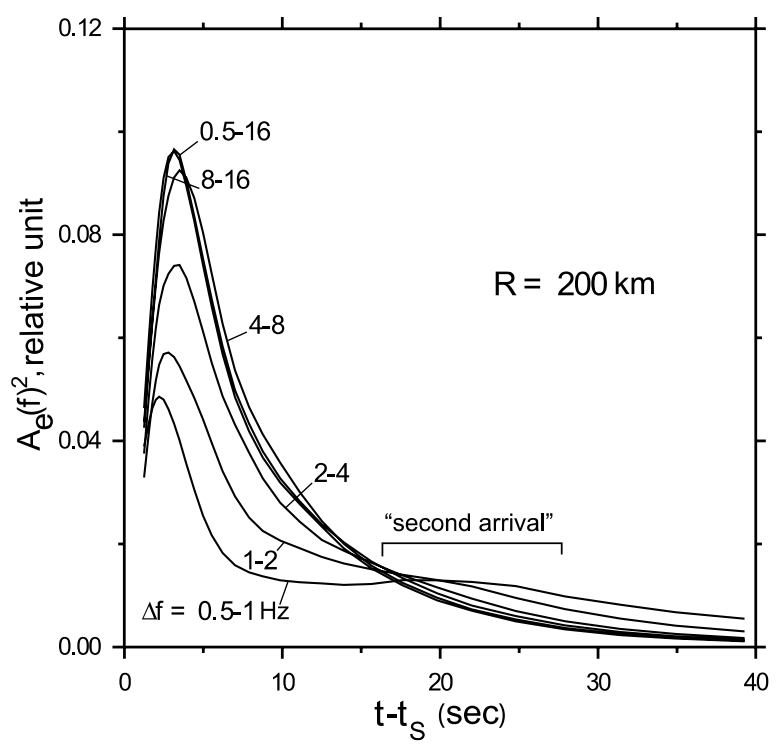

Figure 8

Average smoothed envelopes of the medium pulse response in six frequency bands, reduced to $R=200 \mathrm{~km}$ (linear scale).

Table 2

Estimates of $l$ and $Q_{s}$ for a set of distances and frequency bands

\begin{tabular}{|c|c|c|c|c|c|c|c|}
\hline \multirow{2}{*}{$\begin{array}{c}\text { Freq. Band, } \\
\text { Hz }\end{array}$} & \multirow{2}{*}{$\begin{array}{c}t_{m}(200 \mathrm{~km}) \\
\mathrm{sec}\end{array}$} & \multicolumn{3}{|c|}{$l$ at $R=$} & \multicolumn{3}{|c|}{$Q_{S}$ at $R=$} \\
\hline & & $200 \mathrm{~km}$ & $100 \mathrm{~km}$ & $50 \mathrm{~km}$ & $200 \mathrm{~km}$ & $100 \mathrm{~km}$ & $50 \mathrm{~km}$ \\
\hline $0.5-1$ & 2.3 & 287 & 144 & 72 & 386 & 193 & 97 \\
\hline $1-2$ & 2.7 & 244 & 122 & 61 & 657 & 328 & 164 \\
\hline $2-4$ & 3.4 & 193 & 96 & 48 & 1038 & 519 & 270 \\
\hline $4-8$ & 3.6 & 182 & 91 & 45 & 1960 & 980 & 490 \\
\hline $8-16$ & 3.3 & 199 & 100 & 50 & 4292 & 2146 & 1073 \\
\hline $0.5-16$ & 3.1 & 212 & 106 & 103 & 2476 & 1238 & 619 \\
\hline
\end{tabular}

radiated by a point source in a scattering medium with Gaussian autocorrelation function (ACF), see detailed discussion in GuSEv and ABUBAKIROv (1999a,b). Let us introduce the following denotations: $l=1 / g$ is "effective" (or "transport") meanfree path, $t_{\mathrm{MFP}}=l / v_{S}=Q_{s} / 2 \pi f$ is transport mean-free time, $t_{Q}=Q_{i} / 2 \pi f$ and $v_{S}$ is $S$-wave velocity. Here $Q_{s}$ and $Q_{i}$ denote scattering $Q$ and intrinsic $Q$, respectively; albedo of the medium is $B=t_{\mathrm{MFP}}^{-1} /\left(t_{Q}^{-1}+t_{\mathrm{MFP}}^{-1}\right)=Q_{s}^{-1} /\left(Q_{i}^{-1}+Q_{s}^{-1}\right)$. Now Williamson's result can be written down with a slight modification as

$$
W(\tau, \rho)=\frac{2 \pi^{2}}{\rho^{2}} \sum_{n=1}^{\infty}(-1)^{n-1} n^{2} \exp \left(\frac{-\pi^{2} n^{2} \tau}{\rho^{2}}\right),
$$


where $\tau$ and $\rho$ are dimensionless time and distance: $\tau=t / t_{\mathrm{MFP}}$, and $\rho=R / l$, respectively, and $t$ or $\tau$ are counted from the arrival time. (The original Williamson formula is based, instead of $l$, on the value of "the mean-square angular dispersion of the ray per unit distance" parameter $D=2 / l$ ).

As was shown, e.g., by GUSEV and AbUbAKIROv (1996), the common assumption of Gaussian ACF is hardly realistic for HF seismic waves in the lithosphere. Random media models with power-law spectrum of inhomogeneities can be employed with considerably more success (GUSEV and LemZikov, 1983, 1985; Wu and AKI, 1985). Among such media (of so named "self-affine" class), an important reference case is that of self-similar random inhomogeneity. In this case, in the 3-D spectrum of fluctuations of velocity,

$$
P(k) \propto k^{-\alpha},
$$

the exponent $\alpha$ equals 3. Media with $\alpha>3$ seem to be reasonable models for lithosphere, and $\alpha=3.5-4$ is a good starting estimate (GUSEV and AbUBAKIROv, 1996). As noted by WU and AKI (1985), one can relate the value of $\alpha$ to the value of the exponent (denote it $\gamma_{0}$ ) in the power-law that relates true backscattering turbidity to frequency: $\gamma_{0}=\alpha-4$. Their result is however decidedly more general, and applicable, e.g., for inverse transport mean-free path $1 / l$. Thus, if

$$
l \propto f^{-\gamma}
$$

then one can estimate $\alpha$ as

$$
\alpha=4-\gamma .
$$

Gusev and Abubakirov (1996) numerically modeled pulse shapes for a set of $\alpha$ values, but their shapes are too rough for direct comparison to data. LAMBERT and RicKETT (1999) calculated theoretical pulse shapes for a self-affine medium with $\alpha=11 / 3=3^{2} / 3$ (famous Kolmogorov spectrum for turbulence); and this value fits in the mentioned range 3.5-4. Thus we used their shapes as a good reference. LAMBERT and RicKeTT (1999) also believe that pulse shapes of Williamson's case are identical to those of the case with $\alpha=4$. Envelopes of Lambert and Rickett do not account for intrinsic loss; to clarify its importance we add the relevant attenuation factor in our calculations.

To carry out the comparison of observed and theoretical shapes we must normalize them in an appropriate way. With this aim, we rescale time and amplitude axes of all envelopes (theoretical as well as observed) so that all pulses have unit amplitude and unit onset-to-peak delay. Figure 9 exhibits the result of such a comparison for five one-octave bands. To illustrate the effect of intrinsic loss we plot the "Kolmogorov" case in two versions: with $B=1$ (no intrinsic loss, $\left.Q_{i}=\infty\right)$ and $B=0.5\left(Q_{i}=Q_{s}\right)$. (In the calculation of attenuation, we use the values of onset-to-peak delay and $Q_{s}$ from Table 2, for the wide-band case.) One can see that the effect of a considerable intrinsic loss is only slight, and can be 

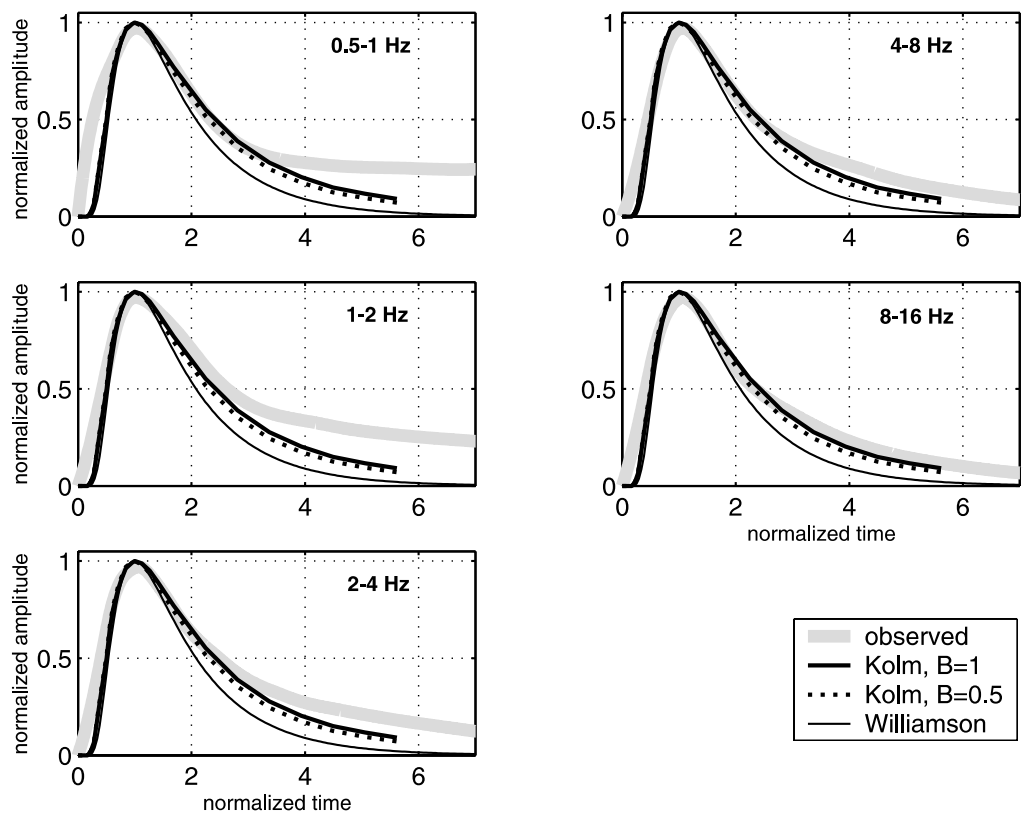

Figure 9

Average Kamchatkan envelope shape for five one-octave bands (gray line), compared to the following theoretical envelope shapes: after WILLIAMSON (1975) for the medium with Gaussian ACF (thin line); after LAMBERT and RICKETT (1999), for the medium with "Kolmogorov" inhomogeneity spectrum (thick lines), in normalized scale. The two versions of "Kolmogorov" envelopes correspond to $B=1.0$ (original shape) and to $B=0.5$ (modified by the present authors). In the vicinity of the peak, the observed shapes are wider than predicted by the Williamson's formula whereas the "Kolmogorov" shape is quite acceptable.

safely ignored for the exploratory study of the present kind. One can note that in the vicinity of the peak, the observed shapes seem to be wider than predicted by the Williamson's formula, whereas the "Kolmogorov" shape is quite acceptable. The leading edge of the observed pulse is poorly resolved, and no meaningful comparison can be made. (The case of $0.5-1 \mathrm{~Hz}$ band is the worst. In this case the duration of a single period of a signal is about $1.4 \mathrm{~s}$, comparable to the value of the peak delay; thus the estimation of the envelope shape cannot be accurate). The picture at the trailing edge (that corresponds to the "early coda" part of a real record) is remarkable. Williamson's shape with its exponentially decaying tail certainly can be rejected as unrealistic. The "Kolmogorov" shape fares well for the $8-16 \mathrm{~Hz}$ band and marginally well for the $4-8 \mathrm{~Hz}$ bands. Numerical results of Gusev and Abubakirov (1996) indicate that the shape of the tail is rather susceptible to the value of $\alpha$. Thus one can believe that the true value of $\alpha$ is near the "Kolmogorov" value of $3^{2} / 3$. At lower frequencies, the agreement between theoretical and observed shapes deteriorates, and becomes worst for the $0.5-1 \mathrm{~Hz}$ band where the already mentioned second hump exists in early coda 
(not seen in Fig. 9). The source of additional energy in the early coda is not settled.

Despite limited agreement between observed and theoretical shapes, we attempted to estimate transport mean-free path values using the values of the onset-to-peak delay $t_{m}$ and ignoring the secondary energy arriving at larger delays. To attain this we follow the approach of ABUBAKIROV and GUSEV (1990), modified by Gusev and Abubakirov (1999a,b). Considering signals within a limited frequency band around a certain $f$ (and omitting the dependence on $f$ in most formulas), we assume for the onset-to-peak delay in dimensionless time $\tau_{m}$

$$
\tau_{m}=C_{m} \rho^{2},
$$

where $C_{m}$ is a model-dependent constant. For the Gaussian ACF/Williamson's case, one can assume $C_{m}=0.091$, and use (14) in the entire $B$ range $0.3-1$ as a reasonable approximation. This gives an estimate

$$
l=C_{m} R^{2} /\left(v_{S} t_{m}\right) .
$$

This quadratic distance dependence holds for multiple low-angle scattering models with finite mean square angle of a single scattering, see GUSEV and ABUBAKIROV (1999a). (In fact, the "low-angle" assumption may be violated in seismological applications, nevertheless we believe that using (15) is a good starting point.) The " finite mean-square angle of a single scattering" property holds for a considerably wide class of scallering models, and in particular includes self-affine media with $\alpha<3.0$ (but not with $\alpha \geq 3.0$ ). For self-affine media, Figure 8 of GuSEV and Abubakirov (1996) permits one to make rough estimates of $C_{m}$ values, valid for $\rho$ values around 1.0. In particular, $C_{m} \approx 0.0315$ for $\alpha=3.0, C_{m} \approx 0.045$ for $\alpha=3.5$, and $C_{m} \approx 0.063$ for $\alpha=4.0$. (These estimates were obtained numerically and do not suffer from the theoretical problem of the "low-angle" approximation mentioned above). Now to apply (15) in the data analysis we must determine $C_{m}$, and to accomplish this we must find $\alpha$ from our observations.

To estimate the exponent $\alpha$ we can use the frequency dependence of $l$, assuming it to follow the power law (12). To find the actual value of $\gamma$ for the Kamchatka crust we note that at a fixed distance and at a certain definite value of $C_{m}$ (that depends only on unknown $\alpha) l(f) \propto t_{m}(f)^{-1}$ (see (15)). We then fit the $t_{m}$ values of Table 2 by a power law, and obtain $\gamma=0.15 \pm 0.05$. Now with (13) we get $\alpha=3.85$. This estimate, retrieved from the frequency dependence of onset-to-peak delay, does not contradict the previous analysis of envelope shapes (that approximately agree with the shape calculated for $\alpha=3.667$ ). For $\alpha=3.85$, interpolation gives $C_{m} \approx 0.057$, and we use this value in further data analysis.

Now employing (15), assuming $v_{S}=3.5 \mathrm{~km} / \mathrm{c}$ and again using observed average delay values $t_{m}$ (Table 2), we obtain the set of estimates of transport mean-free path given in Table 2 for the following distance values: $200 \mathrm{~km}$ (as in Fig. 8) and also 100 and $50 \mathrm{~km}$, see also Figure 10. 


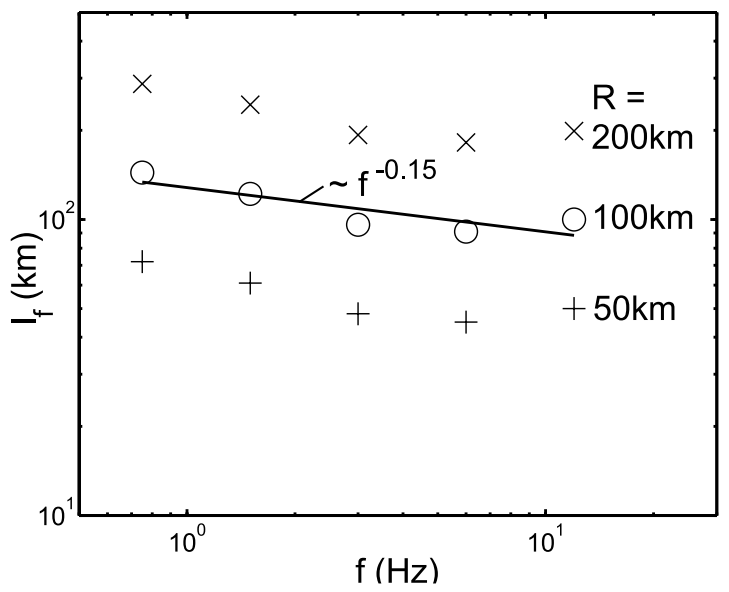

Figure 10

Frequency dependence of the mean free path estimates $l$ (see also Table 2).

Generally speaking, $l$ is a material property, so why do we have such different estimates of $l$ for different distance values $(50,100$ and $200 \mathrm{~km})$ in Table 2? This point needs clarification. First, how did these differences appear from the data? The reason is that empirically the pulse duration grows linearly with distance, whereas the applied theory assumes quadratic dependence. Thus, the estimate of $l$ becomes dependent on distance, and increasing, incidentally, also in linear fashion (instead of being constant, as is appropriate for a regular parameter of the medium). This means that our estimates do not describe the scattering properties of a uniformly scattering medium; rather they are apparent values that reflect the level of scattering along different rays in the nonuniformly scattering medium; more on this point below. The values of dimensionless distance $\rho=R / l$ are in the range $\rho=0.9-1.4$ for the whole distance range analyzed ( $\rho$ being independent of distance and slightly depending on frequency). From the estimates of $l$ one can pass to the values of $Q_{s}=2 \pi f l / v_{S}$ also given in Table 2 .

\section{Stochastic Simulation of Strong Ground Motion}

As we mentioned, the average envelopes can be used for stochastic simulation of strong ground motion records. Broadening of envelopes with distance due to random scattering is especially important for simulating high-frequency seismic records of large, relatively distant, interplate, subduction zone earthquakes. To demonstrate the general methodology of stochastic simulation of strong motions using our average bandpass envelopes we developed a simplified algorithm described briefly in Appendix A. To show it in action we simulated ground motion from two moderate earthquakes recorded by the low-gain (LG) channel of the IRIS station Petropavlovsk: 

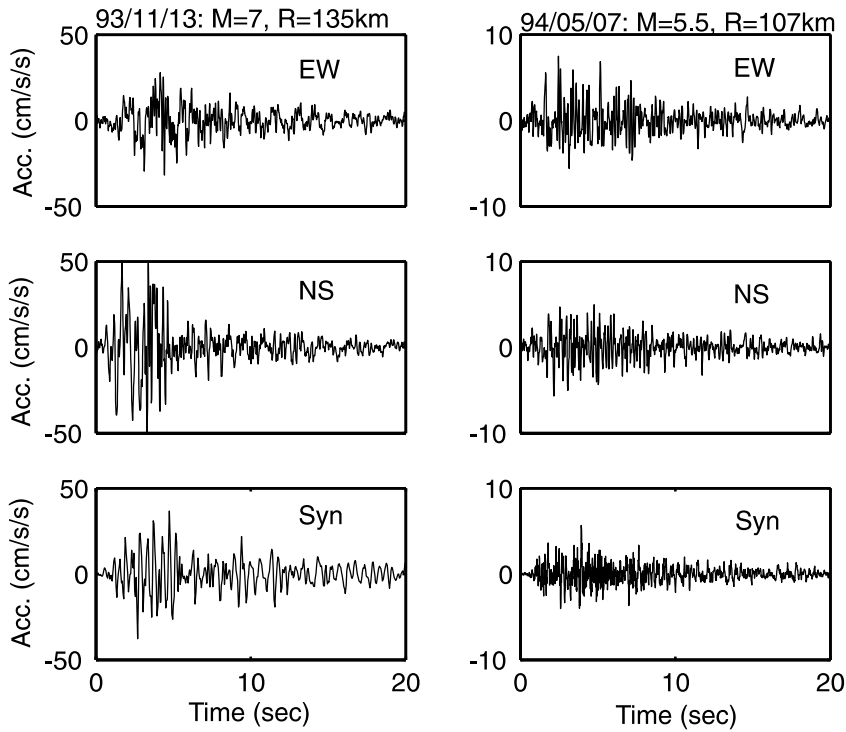

Figure 11

Examples of the synthetic accelerograms (Syn) compared to the observed records (EW and NS components). Left column of subplots shows observed records and synthetic result for magnitude $M=7$ earthquake on November 13,1993, $R=135 \mathrm{~km}$; right column is the same for $M 5.5$ earthquake on May 7 , $1994, R=107 \mathrm{~km}$. Only $S$-wave parts of records were synthesized here.

(1) November 13, 1993 with $M_{W}=7.0, R=135 \mathrm{~km}$, seismic intensity $I=5$ in Petropavlovsk-Kamchatsky, and (2) May 7, 1994, $M_{S}=5.5, R=107 \mathrm{~km}$. On Figure 11 we compare the observed and simulated accelerograms. One can see that both the envelope shape and the frequency content of the observed and simulated records match quite well, despite our use of simplified, average envelopes and spectral scaling law.

\section{Discussion}

When compared to theoretical models of multiple forward scattering, our results are unfavorable. The pulse shape fits marginally well whereas the distance dependence is qualitatively different: linear instead of quadratic. As a possible explanation of the linear trend we propose the propagation of HF energy along curved rays, combined with the fast decay of scattering coefficient with depth (Gusev, 1995; Gusev and Abubakirov, 1999a,b). At short distances, the entire ray lies in the crust, and at long distances, its middle part crosses deeper, less-scattering layers of the earth. As a result, the pulse duration increases with distance, in qualitative agreement with the theory; but the rate of this increase is strongly 
reduced. This effect must be additionally enhanced by the specific properties of scattering in a non-uniformly scattering medium. In this case, the contributions of different segments of the ray to the pulse width are not simply proportional to the length of the segment. According to the relevant formula (see Gusev and AbubaKirov, 1999a), contributions of the end parts of the ray are strongly suppressed, as compared to those of its middle part. Thus, the properties of the medium sampled by this middle part will contribute most significantly to the value of the pulse width.

On the basis of inversion of pulse widths of mostly vertically propagating waves, for the frequencies around $3 \mathrm{~Hz}$, GuSEv and ABubaKirov (1999b) predicted the following apparent $l$ values for the case of shallow earthquakes in the same area: $62 \mathrm{~km}$ for $R=50 \mathrm{~km}, 103 \mathrm{~km}$ for $R=100 \mathrm{~km}$, and $162 \mathrm{~km}$ for $R=300 \mathrm{~km}$, that is, $\rho$ is equal to $1.2,1.0$ and 0.54 , respectively. These results compare quite reasonably to our estimate of $\rho=0.96$ for the $2-4 \mathrm{~Hz}$ band. Very similar results with respect to the true attenuation were obtained by HougH et al. (1988) and TRIFUNAC (1989). They found that the effective whole-path $Q$ values are strongly distance-dependent, analogous to the scattering $Q$ estimates of ourselves. To explain this phenomenon, the same idea is proposed, namely, that with increasing distance rays dive deeper and probe less attenuating layers.

Abubakirov and Gusev (1990) estimated $l$ of $S$-waves for the station PET from the ratio of amplitudes between direct wave and coda both having the same travel time, (namely $t_{c}=25 \mathrm{sec}$ ), thus excluding the bias related to the unknown $Q_{i}$ value. In their interpretation they used theoretical asymptotic coda obtained for the primitive isotropic scattering model, and obtained $l$ estimates ranging from 70 $140 \mathrm{~km}$ for the frequency band 1-8 Hz. However the numerical modeling of GusEv and Abubakirov (1996) for more realistic forward-scattering cases (Figs. 5 and 6 in their paper) revealed that the assumed theoretical coda level was about twice the accurate one. To incorporate this adjustment, the mentioned $l$ estimates must be halved, giving the range $l=35-70 \mathrm{~km}$. To find the distance range to which these results pertain, we note that in the simplest single-scattering coda model the coda lapse time of $t_{c}=25 \mathrm{sec}$ corresponds to a one-way travel distance of $25 * 3.5 / 2=44 \mathrm{~km}$. Subsequently, the range $l=35-70 \mathrm{~km}$ from the relative coda level can be compared to the results of Table 2 for the distance of $50 \mathrm{~km}$ that show the range $l=45-61 \mathrm{~km}$ for the same frequency band. The match is quite acceptable.

The additional energy in the early coda in the frequency range $0.3-2 \mathrm{~Hz}$ (see Fig. 8, 15-30 s range) was mentioned by many researchers (e.g., RAUTIAN et al., 1981) and generally ascribed to trapped short-period surface waves in sedimentary wave guides. No geological features with such a signature are known around Petropavlovsk-Kamchatsky. This point deserves more detailed study.

The general fit of HF envelope shapes in the $S$-wave group to the models of forward scattering (developed for uniformly scattering medium) is quite reasonable, in contrast with the trend for duration that is poor. To model more consistently the 
properties of observed envelopes, one should pass to more advanced, first of all layered, models of the scattering medium.

\section{Conclusions}

A new approach to the analysis of the duration parameters and to the determination of empirical average envelopes of small earthquakes has been successfully tested. The results of the analysis have been presented in a form ready for implementation into algorithms for the simulation of strong ground motion earthquakes.

For each frequency band studied we established that the path-related duration parameter $T_{\mathrm{rms}}$ increases linearly with distance for shallow Kamchatka earthquakes between $R=30$ and $R=200 \mathrm{~km}$, in agreement with similar trends found earlier for other regions. The quadratic trend predicted for forward-scattered body waves in the uniformly scattering medium can be rejected with certainty. The envelope shapes have been estimated for all frequency bands covering the $0.5-16 \mathrm{~Hz}$ range. For higher-frequency bands these shapes closely remind those of the forward-scattered body-wave pulse. For lower-frequency bands the envelopes may be tentatively interpreted as the combination of a similar scattered pulse and of a train of HF surface waves.

In this study we determined average values of $T_{\mathrm{rms}}$, average signal envelopes of the horizontal component, and parameters of dispersion for both. This material represents a solid basis for stochastic simulation of strong-motion earthquakes on Eastern Kamchatka, providing an advanced description of the path-related contribution to the signal envelope from a large earthquake.

We also estimated parameters of apparent scattering attenuation for nearhorizontal seismic wave propagation in the crust of Kamchatka.

\section{Acknowledgements}

The authors are grateful to V.V. Yashuk and D.V. Droznin who helped to extract digital data. Advice and comments of I.R. Abubakirov were highly valuable. He also referred to us the paper of LAMBERT and RICKETT (1999). The authors are indebted to Axel Plešinger and to Iwan Pšenčik for comments that enhanced the paper.

\section{Appendix A: Algorithm for Stochastic Simulation of Accelerograms}

The proposed algorithm can be used to synthesize high-frequency strong ground motion records (accelerograms) of relatively distant $(R \sim 70-200 \mathrm{~km})$ earthquakes 
with magnitudes $M<7-7.5$. At these distances and in the frequency band $0.5-20 \mathrm{~Hz}$ we can assume that the properties of strong motion will be defined by random scattering of S waves. A synthetic record is obtained from the sum of the set of oneoctave bandpass stochastic accelerograms (for frequency bands $0.5-1,1-2,2-4,4-8$ and $8-16 \mathrm{~Hz}$ ). Each bandpass stochastic accelerogram is calculated as modulated random noise with the envelope and spectrum specific for a particular source-site combination. In many aspects, the algorithm reproduces the approaches of RAUTIAN (1976) and BOORE (1983).

\section{A.1. General Outline of the Algorithm}

The flow chart in Figure 12 illustrates the principal relationship between the initial, intermediate and output quantities of the algorithm. The main input parameters are the moment magnitude $M_{W}$ [or seismic moment $\left.M_{0} ; \log M_{0}=1.5\left(M_{W}+10.7\right)\right]$ and the hypocentral distance $R$. For these parameters, and for each frequency band, the algorithm calculates: (1) the mean bandpass envelope and (2) the amplitude Fourier spectrum of the accelerogram. The Fourier spectrum is used to generate a realization of stationary random noise with the spectral shape similar to the spectral shape of the expected earthquake record. An octave-band synthetic record is calculated by multiplying noise and envelope amplitudes:

$$
A_{s}(t)=A_{e}(t) \cdot A_{n}(t),
$$

where $A_{s}(t)$ is the synthesized accelerogram, $A_{e}(t)$ is the rms amplitude of the envelope, and $A_{n}(t)$ is the time history of the noise. Then, octave-band records for all frequency bands are added to produce the complete synthetic accelerogram. Such an approach enables us to simulate records with both nonstationary amplitude and nonstationary carrier frequency content.

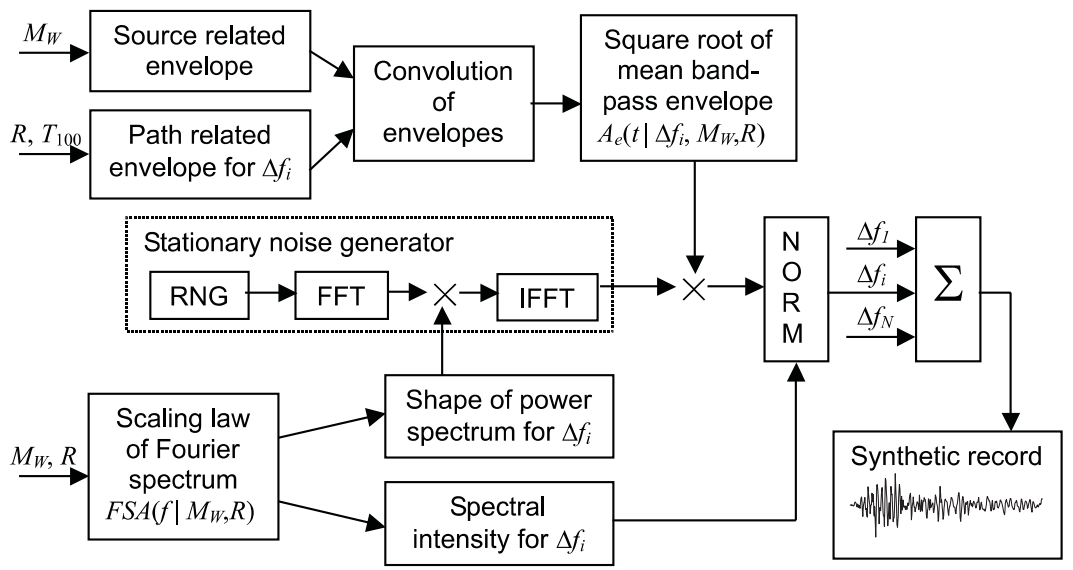

Figure 12

The flow-chart of the algorithm for the stochastic simulation of accelerograms. 


\section{A.2. Mean Bandpass Envelope}

We presume that the envelope of an accelerogram $A_{e}^{2}(t)$ can be calculated by convolution of the source-related envelope $A_{s e}^{2}(t)$ and the path-related envelope $A_{p e}^{2}(t)$ :

$$
A_{e}^{2}(t)=A_{s e}^{2}(t) * A_{p e}^{2}(t) .
$$

This is true for the case of point seismic source and we extend this approach for the case of a finite size source of radiation recorded at a large distance. Sometimes the third component may be significant, namely the site-related envelope, in particular for non-rock sites with complicated topography, nonetheless the present version of the algorithm ignores this possibility.

The source-related envelope $A_{s e}(t)$ is assumed to have a trapezoid shape whose duration is defined by the value of the moment magnitude. Following GuSEV and Melnikova (1990) we set the duration of this trapezoid ("source duration", defined by the $50 \%$ level of maximum amplitude) according to the following simple relationships that assume self-similarity:

$$
T_{s}=L_{s} / v_{s}
$$

and

$$
\lg L_{s}=0.5 M_{W}-C_{L}+a .
$$

Here $T_{s}$ is the source duration, $L_{s}$ is the source length, $v_{s}$ is the rupture velocity, $C_{L}$ is an empirical "universal" constant and $a$ is a constant correction term for a particular source (related to effects of directivity, stress drop etc. usually $a=0$ ). For Kamchatka subduction zone earthquakes we can assume $C_{L}=1.85$ and $v_{s}=3.5 \mathrm{~km} / \mathrm{s}$. A relatively high assumed value of rupture velocity reflects the fact that the HF source radiation envelope is usually a series of pulses with a total duration shorter than the LF duration (HARTZELL and Heaton, 1985). The sourcerelated envelopes are the same for all frequency bands.

The path-related envelopes, $A_{p e}\left(t \mid \Delta f_{i}\right)$, are frequency dependent; for a particular distance $R$ they are calculated directly from the shapes of average envelopes of Figure 8 , with the time scale compressed according to the value of the distance $R$.

\section{A.3. Calculation of Fourier Spectrum}

The Fourier spectrum of the accelerogram, FSA, of an earthquake with magnitude $M_{W}$ recorded at a rock site at a hypocentral distance $R$, is calculated according to the average empirical Fourier spectrum scaling law, $F S A\left(f \mid M_{W}, R\right.$, rock) for Kamchatka (Petukhin et al., 1999). This scaling law accounts for: (1) nonlinear magnitude dependence (saturation of HF radiation at large magnitudes $M_{W}$ ), (2) $f_{\max }{ }^{-}$ phenomenon with magnitude dependent $f_{\max }$ - value ( $f_{\max }$ decreasing with increasing 
magnitude), (3) spherical geometrical spreading and anelastic attenuation ( $Q$-value) during the wave propagation, (4) saturation of amplitudes near a source of a finite size; it also incorporates the specific spectral station correction for station Petropavlovsk (PET) whose records were simulated.

\section{A.4. Stationary Noise Generator}

A realization of stationary noise $A_{n}(t)$ with proper spectral properties is generated as follows: (1) Using a random number generator (RNG in Fig. 12) a series of $N$ random numbers ( $N$ - number of counts of simulated accelerogram) is generated; this series represents a realization of random "white" noise. (2) The series is Fourier transformed (FFT). (3) The amplitude Fourier spectrum of the realization is multiplied by the bandpassed FSA of the accelerogram. (4) Inverse Fourier transformation yields a realization of random noise whose spectrum is proportional to the spectrum of the simulated accelerogram.

\section{A.5. Calculation of Synthetic Accelerogram}

The next step is traditional in stochastic simulation of accelerograms: the mean bandpass amplitude envelope, $A_{e}\left(t \mid \Delta f_{i}, M_{W}, R\right)$, is multiplied by the calculated realization of the random noise, $A_{n}$, according to equation (A.1). If the envelope duration is small compared to the inverse value of the mean frequency of random noise, such multiplication may distort (broaden) the spectrum of the noise. In most practical cases, such a distortion is negligible; in any case, its absence was checked in the procedure.

At this step the synthesized bandpass record has proper spectral and envelope shapes, although its amplitude level is still arbitrary. To set this level properly, we calibrate the synthesized record using the spectral power of the initial Fourier spectrum integrated over an appropriate spectral band (block NORM in Fig. 12).

We repeat the described calculations for each one-octave frequency band $(0.5-1$, $1-2,2-4,4-8$ and $8-16 \mathrm{~Hz}$ ) and obtain the final synthetic accelerogram by summing up all bandpass simulated accelerograms.

If the information regarding the size and location of subsources (asperities) is available, the algorithm can be modified for cases of $M>7.5$ and for near-fault sites.

\section{REFERENCES}

Abubakirov, I. R. and Gusev, A. A. (1990), Estimation of Scattering Properties of Lithosphere of Kamchatka Based on Monte-Carlo Simulation of Record Envelope of a Near Earthquake, Phys. Earth. Planet. Inter. 64, 52-67.

APTIKAEv, F. F., The bearing of the duration of seismic oscillations on instrumental evaluation of seismic intensity, In Seismicheskaya shkala I metody izucheniya seismicheskoi intensivnosti (Nauka, Moscow, 1975) pp. 234-239 (in Russian). 
Boore, D. M. (1983), Stochastic Simulation of High-frequency Ground Motions Based on Seismological Models of the Radiated Spectra, Bull. Seismol. Soc. Am. 73, 1865-1894.

Gusev, A. A. (1983), Descriptive Statistical Model of Earthquake Source Radiation and its Application to an Estimation of Short-Period Strong Motion, Geophys. J. R. astr. Soc. 74, 787-808.

Gusev, A. A. (1995), Vertical Profile of Turbidity and Coda Q, Geophys. J. Int. 123, 665-672.

Gusev, A. A. and Abubakirov, I. R. (1996), Simulated Envelopes of Non-isotropically Scattered Body Waves as Compared to Observed Ones: Another Manifestation of Fractal Heterogeneity, Geophys. J. Int. 127, 49-60.

Gusev, A. A. and Abubakirov, I. R. (1999a), Vertical Profile of Effective Turbidity Reconstructed from Broadening of Incoherent Body-wave Pulses-I. General Approach and the Inversion Procedure, Geophys. J. Int. 136, 295-308.

Gusev, A. A. and Abubakirov, I. R. (1999b), Vertical Profile of Effective Turbidity Reconstructed from Broadening of Incoherent Body-wave Pulses-II. Application to Kamchatkan Data, Geophys. J. Int. 136, 309-323.

Gusev, A. A. and Lemzikov, V. K. (1983), Estimation of Scattering Parameters of Shear Waves in the Crust and Upper Mantle of Kamchatka According to Observations of "Shipunski" Station, Volc. Seis. \#1, 94-108.

Gusev, A. A. and Lemzikov, V. K. (1985), Properties of Scattered Elastic Waves in the Lithosphere of Kamchatka: Parameters and Temporal Variations, Tectonophysics 112, 137-153.

Gusev, A. A. and Melnikova, V. N. (1990) Relations between Magnitudes: Global and Kamchatka Data, Vulkanologiya i Seismologiya 6, 55-63 (in Russian).

Haddon, R. A.W. (2000), Comment on "Evaluation of Models for Earthquake Source Spectra in Eastern North America" by Gail M. Atkinson and David M. Boore, Bull. Seismol. Soc. Am. 90, 1332-1338.

Hartzell, S. H. and Heaton, T. N. (1985), Teleseismic Time Functions for Large, Shallow Subduction Zone Earthquakes, Bull. Seismol. Soc. Am. 75, 965-1004.

Hough, S. E., Anderson, J. G., Brune, J., Vernon III, F., Berger, J., Fletcher, J., HaAr, L., Hanks, T., and BAKer, L. (1988), Attenuation near Anza, California, Bull. Seismol. Soc. Am. 88, 672-691.

Ishimaru, A., Wave Propagation and Scattering in Random Media (Academic Press, New York 1978).

Kamae, K., Irikura, K., and Pitarka, A. (1998), A Technique for Simulating Strong Ground Motion Using Hybrid Green's Function, Bull. Seismol. Soc. Am. 88, 357-367.

Kawashima, K., Aizawa, K., and Takahashi, K., Duration of strong motion acceleration records, In Proc. of JSCE Structural Eng./Earthquake Eng. (1985) 2, 2.

Kopnichev, Yu. F. and ShPIlker, G. L. (1978), Parameters of High-frequency Radiation of Earthquake Source and Strong-motion Simulation, Doklady Acad. Nauk 239, 193-196 (in Russian).

Lambert, H. C. and Rickett, B. J. (1999), On the Theory of Pulse Propagation and Two-frequency Field Statistics in Irregular Interstellar Plasmas, Astrophys. J. 517, 299-317.

Leary, P., Igel, H., Mora, P., and Rodrigues, D. (1993), Finite-difference Simulation of Trapped Wave Propagation in Fracture Anisotropic Low-velocity Layers, Can. J. Explor. Geophys. 29, 31-40.

Malin, P. E. (1980), A First-order Scattering Solution for Modeling Elastic Wave Codas; I. The Acoustic Case, Geophys. J. Roy. Astron. Soc. 63, 361-380.

Petukhin, A. G., Gusev, A. A., Guseva, E. M., Gordeev, E. I., and Chebrov, V. N. (1999), Preliminary Model for Scaling of Fourier Spectra of Strong Ground Motion Recorded on Kamchatka, Pure Appl. Geophys. 156, 445-468.

RAUtian, T. G., The role of source function and medium response in the model of generation of seismic motion, In Voprosy injenernoi seismologii (Nauka, Moscow, 1976) 18, pp. 3-14 (in Russian).

Rautian, T. G. and Nikiforova, M. M., A study of the spectral and temporal characterisitics of earthquakes in the vicinity of dam of the Nurek hydroelectric power plant, In Seismic Effect on Hydrotechnical and Power Plant Construction (Nauka, Moscow 1980) pp. 144-158 (in Russian).

Rautian, T. G., Khalturin, V. I., and Zakirov, M. S., Experimental'nye issledovaniya seismicheskoi kody (Nauka, Noscow 1981, in Russian).

SAto, H. (1989), Broadening of Seismogram Envelopes in the Random Inhomogeneous Lithosphere Based on the Parabolic Approximation: Southeastern Honshyu, Japan, J. Geophys. Res. 94, 17,735-17,747. 
TrifunAC, M. D. (1989), Empirical Scaling of Fourier Spectrum Amplitudes of Recorded Strong Earthquake Accelerations in Terms of Magnitude and Local Soil and Geologic Conditions, Earthq. Engin. Engin. Vibration 9, 23-44.

Trifunac, M. D. and Brady, A. G. (1975), A Study of the Duration of Strong Earthquake Ground motion, Bull. Seismol. Soc. Am. 65, 581-626.

Wang, Ch. Y. and Herrmann, R. B. (1988), Synthesis of Coda Waves in Layered Medium, Pure Appl. Geophys. 128, 7-42.

Williamson, I. P. (1972), Pulse Broadening Due to Multiple Scattering in the Interstellar Medium, Mon. Not. R. Astr. Soc. 157, 55-71.

Williamson, I. P. (1975), The Broadening of Pulses Due to Multipath Propagation of Radiation, Proc. R. Soc. Lond. A 343, 131-147.

Wu, R.-S. and AKI, K. (1985), The Fractal Nature of the Inhomogenneities in the Lithosphere Evidenced from Seismic Wave Scattering, Pure Appl. Geophys. 123, 805-818.

(Received October 23, 2001, accepted March 10, 2002)

\section{(20) To access this journal online: \\ (4P) http://www.birkhauser.ch}

